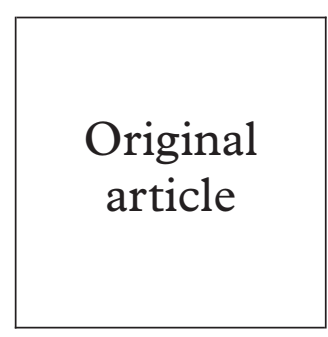

\title{
Partner notification for gonorrhoea: a comparative study with a provincial and a metropolitan UK clinic
}

\author{
Gill Bell, Helen Ward, Sophie Day, Azra C Ghani, Una Goan, Elizabeth Claydon, \\ George R Kinghorn
}

Objective: To compare partner notification practice and outcomes at a provincial and a metropolitan clinic.

Design: Prospective study, following standardisation of partner notification policy.

Settings: Sheffield Department of Genitourinary Medicine, Royal Hallamshire Hospital and Jefferiss Wing Centre for Sexual Health, St Mary's Hospital, London.

Subjects: Consecutive patients with culture positive gonorrhoea between October 1994 and March 1996 who were interviewed by a health adviser.

Results: In Sheffield, 235 cases reported 659 outstanding contacts, of whom 129 (20\%) were subsequently screened, and $65(50 \%)$ had gonorrhoea. At St Mary's 510 cases reported 2176 outstanding contacts, of whom 98 (5\%) were known to have been screened, and $53(54 \%)$ had gonorrhoea. Patient or provider referral agreements appeared more productive in Sheffield, where $60 \%$ resulted in contact attendance, compared with $13 \%$ at St Mary's. Provider referral was used more frequently in Sheffield, for $44 \%$ of referrals, compared with $1 \%$ at St Mary's. Multivariate analysis showed that partner notification was less effective for casual and short term $(<7$ days $)$ partnerships in both centres, and for homosexual men at St Mary's.

Conclusion: Partner notification outcomes were better in the provincial setting where contact attendance could be recorded more reliably and provider referral was used more extensively. The high proportion of contacts who remained untraced in both settings indicates the need for complementary screening and prevention initiatives.

(Sex Transm Inf 1998;74:409-414)

Keywords: partner notification; contact tracing; gonorrhoea

\section{Introduction}

Partner notification contributes to the control of gonorrhoea by identifying and treating previously undiagnosed infection in the sexual contacts of known cases, thereby preventing reinfection of the index patient, or transmission to others. The effectiveness of the strategy depends upon the ability of the patient or health adviser to locate contacts and ensure they attend for screening. Determining the success of partner notification requires a reliable method of verifying contact attendance. These factors may be influenced by local conditions, including the sexual behaviour and mobility of the population and local service provision.

Recent studies have indicated that the process and outcome of partner notification are influenced by clinic size, location, and HIV workload. ${ }^{1-3}$ A national UK gonorrhoea management survey ${ }^{1}$ found that the number of contacts per case attending big London clinics was half the number attending small provincial services. A survey of work roles in genitourinary medicine clinics ${ }^{2}$ indicated that the partner notification process was challenged in large metropolitan clinics by a diversion of health adviser resources towards HIV related counselling, and by an associated shift in emphasis from public health to individual patient care.
To explore the relation between local conditions, partner notification practice, and outcomes we have undertaken a comparative study with two clinics which differed significantly in terms of location and workload. This research was carried out within a larger study of gonorrhoea transmission networks. ${ }^{4}$

\section{Comparison of study sites}

LOCATION AND POPULATION

The Department of Genitourinary Medicine in Sheffield is the only clinic serving the stable, largely indigenous population of this provincial city. Approximately $95 \%$ of attendees are local residents, who would need to travel more than 10 miles to access an alternative service. The Jefferiss Wing Centre for Sexual Health at St Mary's Hospital, London, is one of several clinics serving the more mobile cosmopolitan population of west London. Proximity to tube and mainline stations makes the clinic accessible to the higher proportion of attendees who live outside the area. There are 15 alternative services within a 10 mile radius.

\section{WORKLOAD AND RESOURCES}

Internal records for 1995 showed a smaller overall workload in Sheffield, where 13049 diagnostic episodes were recorded compared with 30627 at St Mary's. The incidence of gonorrhoea per 100 attendees was comparable between services, at 2.3 (95\% CI 2.0-2.6) for 
Sheffield and 2.7 (95\% CI 2.5-3.0) for St Mary's. The HIV related workload was smaller in Sheffield, where the prevalence of HIV per 100 attendees was 0.15 (95\% CI 0.06-0.23) compared with 6.29 at St Mary's (95\% CI 5.93-6.63).

Health advisers responsible for partner notification had proportionally more resources in Sheffield during the study period. Five health advisers were available for 249 cases of gonorrhoea (49.8 per health adviser), with access to three interview rooms in Sheffield. In contrast, at St Mary's seven health advisers offered a service for 648 cases of gonorrhoea (92.6 per health adviser) with access to two interview rooms. During the study period the Sheffield health advising team remained stable, while St Mary's trained six new members. In both settings health advisers undertook HIV related counselling. The greater workload at St Mary's was shared with other clinic staff and supported by a comprehensive range of voluntary services.

\section{PARTNER NOTIFICATION POLICY}

Before the study, partner notification practice had been more intensive in Sheffield, where case patients were offered the choice of notifying contacts themselves (patient referral) with the use of a contact slip, or allowing the health adviser to do so without mentioning their name (provider referral). Name, age or date of birth, and address were sought for each contact to enable attendance to be verified, and to facilitate provider referral. Partial information was supplemented from local health records for tracing purposes. These included the Patient Administration System (PAS), which stores names and addresses of all hospital trust attendees, and the Family Health Services Authority (FHSA), which was authorised to release the name of a contact's general practitioner who could then supply a current address. Confidentiality was maintained when liaising with general practitioner surgeries, which were informed that the details were sought by an unspecified hospital department. Case patients were reinterviewed in Sheffield unless partner notification had been resolved, and verification of contact attendance was routinely sought from in house records or other services. Before the study at St Mary's, partner notification activities had been restricted to one interview, during which patient referral was encouraged and contact slips issued: data collection, provider referral, and verification of contact attendance had not formed part of routine practice.

\section{CLINICAL MANAGEMENT}

Before and during the study, clinical management of gonorrhoea differed between centres in relation to epidemiological prescribing policy and test of cure recommendations. In Sheffield, epidemiological treatment for chlamydia was deferred until the first test of cure at 3 days and repeat tests at 2 weeks were advised. Known contacts with negative gonorrhoea microscopy were not routinely treated epidemiologically but were asked to return in 3 days for culture results and repeat tests. Exceptions to deferred treatment were made for contacts who were not expected to return. Untreated defaulters were contacted by a health adviser. At St Mary's gonorrhoea treatment and anti-chlamydial therapy were prescribed concurrently on the day of presentation to all cases and known contacts. One test of cure at 2 weeks was recommended.

\section{Methods}

All cases of culture positive gonorrhoea diagnosed between October 1994 and March 1996 were eligible for the study and were included providing they had been interviewed by a health adviser. Referral of cases to a health adviser was established policy in both clinics and was prompted by a written reminder in the patient notes.

STANDARDISATION OF POLICY

Partner notification policies were standardised as far as possible during the study period. St Mary's adopted the key elements of the more intensive Sheffield policy including contact data collection, provider referral, follow up case interviews and verification of contact attendance. Standardisation was incomplete because Sheffield continued to follow procedures which were not adopted by St Mary's, including the use of local health records, and the follow up of partner notification issues with cases who had ceased to attend. St Mary's did not negotiate access to local PAS and FHSA records because contacts were less likely to be registered locally given the mobility of the population and the proportion of cases from outside the immediate area.

Prescribing policies were not standardised because deferred epidemiological treatment carries a risk of increased complications and onward transmission: this risk may be greater within a mobile metropolitan population where defaulters are more difficult to trace.

DATA COLLECTION AND RECORDING

Data for partner notification outcomes were collected prospectively during health adviser interview. Patients were asked to indicate the total number of individuals with whom they had had protected or unprotected vaginal, oral, or anal contact during the previous 3 months. Identifying information was sought for each contact, including name, date of birth or age, and address where available. Patient or provider referral was negotiated for those contacts who were thought to be unscreened, at risk of infection, and potentially traceable. Standardised study forms were used to record case and contact demographics, relationship characteristics, date of screening, and gonorrhoea diagnosis. The partner notification agreement for each contact (already attended; patient to notify; health adviser to notify; patient refuses; untraceable; other) was recorded and updated at each interview.

DEFINITIONS AND CLASSIFICATIONS

All episodes of culture positive gonorrhoea were classified as cases, including those in indi- 
viduals who had presented as contacts. Repeat infections were counted as further cases if sexual history suggested reinfection rather than treatment failure. For the purpose of comparison all sexual partners were counted as contacts, including those who were not actively sought because they were untraceable, or were unlikely to have been involved in transmission. Individuals named by more than one case were counted as a contact more than once, and contacts who were positive for gonorrhoea were counted both as cases and as contacts. ${ }^{5}$ Clinic attendees who identified themselves as contacts of cases but who were not named or indicated by the case during interview were excluded from the calculations. (Sheffield $\mathrm{n}=20$; St Mary's $\mathrm{n}=0$ ). Contacts were classified as screened if verified attendance occurred within 90 days of the case diagnostic test. We differentiated between contacts who did not require notification because they had already been screened, and outstanding contacts, who were the potential target of the intervention (even though many were not actively sought).

OUTCOME MEASURES

There is no single standard outcome measure which adequately evaluates the range of objectives of partner notification. The measures used here have been selected for their aggregate ability to provide comprehensive assessment.

The ultimate aim of partner notification is to reduce transmission of gonorrhoea in the population by securing the treatment of an unknown number of infected contacts. Measures used to evaluate effectiveness include the proportion of outstanding contacts screened, the proportion of cases with at least one contact attending, and the proportion of cases linked to another case.

The working goal of partner notification is to secure the attendance of as many potentially traceable contacts as possible through negotiated patient or provider referral. We used the proportion of referral agreements which re-

Sheffield

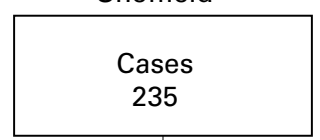

\section{Contacts previous}

3 months 768

3.3 per case sulted in contact attendance to measure the productivity of staff efforts. The uptake and outcome of provider referral were also assessed separately to evaluate the usefulness of a contact tracing service.

\section{STATISTICAL ANALYSIS}

Each study participant was allocated a unique number, and standardised data were entered onto a relational database constructed in ACCESS. Statistical analyses were undertaken using SAS. ${ }^{6}$ Univariate analyses and logistic regression were used to assess the effect of case demographics (sex, sexual preference, age, ethnicity, number of sexual partners) and partnership characteristics (type and duration) on contact attendance. Two outcome measures were used-whether the contact attended, and whether the contact was culture positive for gonorrhoea. Observations are not independent since cases may report more than one contact, and contacts may also occur as cases (although in the latter case duplicate partnership observations do not result since we only considered outstanding contacts). Observations may therefore be correlated if they form part of the same cluster of connected individuals in the sexual partner network. Generalised estimating equations ${ }^{7}$ were therefore used to determine robust confidence intervals and $p$ values in both the univariate analyses and the logistic regression, assuming unknown correlation within clusters. As the type and duration of partnership are highly correlated, two separate regression models were assessed to avoid the effects of multi-colinearity (estimates for the case demographics are presented from the model with partnership type only as similar results were obtained from the model with partnership duration). In general there were insufficient data points to consider first and higher order interactions.
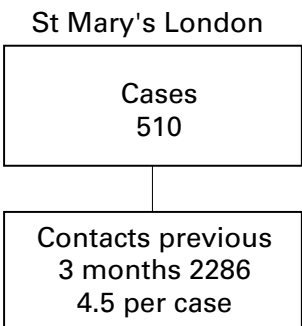

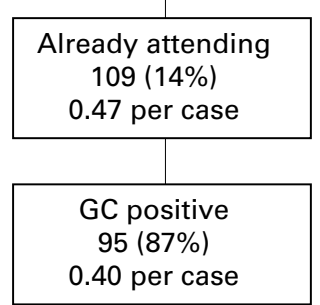

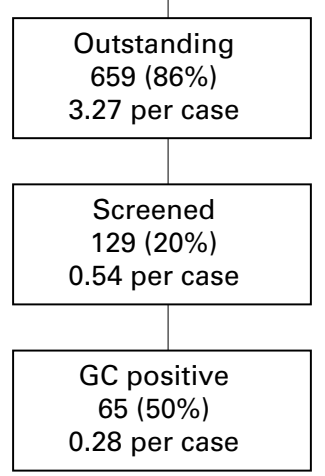

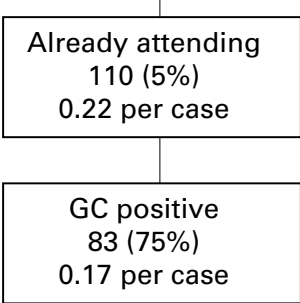

0.10 per cas

Figure 1 Partner notification outcomes. 


\section{Results}

STUDY POPULATION

The study included 235 cases in Sheffield and 510 cases at St Mary's. A detailed description and demographic comparison of the two samples is reported elsewhere. ${ }^{4}$ A greater proportion of the St Mary's population were homosexual or bisexual males, and people of non-white ethnicity and non-British nationality. The proportion of gonorrhoea cases interviewed by a health adviser for inclusion in the study was higher in Sheffield than St Mary's $(94 \%$ v $79 \%$, p <0.001). Female cases were more likely to have been missed in Sheffield $\left(\chi^{2}=12.6, \mathrm{p}<0.001\right)$ whereas male cases were more likely to have been missed at St Mary's $\left(\chi^{2}=6.4, p=0.01\right)$.

NUMBERS OF CONTACTS

The number of contacts reported was 768 in Sheffield (median 2, range $1-40$ ) and 2286 at St Mary's (median 2, range 0-150). Standardised

Table 1 Effect of patient demographics and relationship details on partner notification outcome (a) in Sheffield and (b) at St Mary's

\begin{tabular}{|c|c|c|c|c|c|c|}
\hline & \multicolumn{3}{|c|}{ Outstanding contact attendance } & \multicolumn{3}{|c|}{ Outstanding contact GC positive } \\
\hline & Univariate $O R$ & $\begin{array}{l}\text { Multivariate } \\
\text { adjusted OR }\end{array}$ & p Value & Univariate $O R$ & $\begin{array}{l}\text { Multivariate } \\
\text { adjusted OR }\end{array}$ & $p$ Value \\
\hline \multicolumn{7}{|l|}{ (a) Sheffield } \\
\hline \multicolumn{7}{|l|}{ Individuals } \\
\hline \multicolumn{7}{|l|}{ Sex } \\
\hline male & 1.0 & 1.0 & - & 1.0 & 1.0 & - \\
\hline female & $1.1(0.6,1.9)$ & $0.6(0.3,1.5)$ & 0.30 & $0.4(0.2,0.8)$ & $0.3(0.1,0.7)$ & 0.01 \\
\hline \multicolumn{7}{|l|}{ Orientation } \\
\hline heterosexual & 1.0 & 1.0 & - & 1.0 & 1.0 & - \\
\hline homosexual & $0.3(0.2,0.6)$ & $0.4(0.1,1.4)$ & 0.16 & $0.4(0.2,1.0)$ & $0.5(0.1,1.6)$ & 0.23 \\
\hline \multicolumn{7}{|l|}{ Age group } \\
\hline under 20 & 1.0 & 1.0 & - & 1.0 & 1.0 & - \\
\hline $20-25$ & $1.2(0.6,2.4)$ & $1.8(0.7,4.7)$ & 0.21 & $0.5(0.2,0.95)$ & $0.5(0.2,1.2)$ & 0.11 \\
\hline $25-30$ & $1.2(0.6,2.4)$ & $1.5(0.6,3.8)$ & 0.34 & $1.0(0.4,2.2)$ & $0.8(0.3,2.1)$ & 0.61 \\
\hline over 30 & $1.4(0.7,2.9)$ & $1.6(0.6,4.4)$ & 0.35 & $1.0(0.5,2.4)$ & $0.8(0.3,2.4)$ & 0.73 \\
\hline missing & & $3.9(0.2,83.9)$ & 0.39 & $1.9(0.1,32.2)$ & $2.7(0.1,58.5)$ & 0.54 \\
\hline \multicolumn{7}{|l|}{ Ethnicity } \\
\hline white & 1.0 & 1.0 & - & 1.0 & 1.0 & - \\
\hline black & $1.7(0.9,3.1)$ & $1.1(0.5,2.5)$ & 0.88 & $2.6(1.4,4.9)$ & $1.3(0.5,3.1)$ & 0.56 \\
\hline other & $1.3(0.4,4.5)$ & $0.6(0.1,3.4)$ & 0.59 & $1.6(0.3,8.6)$ & $1.0(0.2,6.3)$ & 0.97 \\
\hline missing & $1.1(0.3,4.1)$ & $1.1(0.1,11.0)$ & 0.96 & $2.0(0.8,5.0)$ & $1.4(0.2,10.8)$ & 0.65 \\
\hline No of partners & $0.9(0.9,1.0)$ & $1.0(0.9,1.1)$ & 0.44 & $0.95(0.85,1.05)$ & $1.0(0.9,1.1)$ & 0.87 \\
\hline \multicolumn{7}{|l|}{ Partnerships } \\
\hline \multicolumn{7}{|l|}{ Type } \\
\hline regular & 1.0 & 1.0 & - & 1.0 & 1.0 & - \\
\hline casual & $0.3(0.2,0.5)$ & $0.3(0.2,0.5)$ & $<0.001$ & $0.4(0.2,0.8)$ & $0.4(0.2,0.7)$ & 0.001 \\
\hline other & $1.7(0.6,4.8)$ & $1.5(0.4,5.2)$ & 0.53 & $0.4(0.1,1.7)$ & $0.3(0.1,1.3)$ & 0.10 \\
\hline missing & $0.2(0.1,0.6)$ & $0.2(0.1,0.5)$ & $<0.001$ & $0.4(0.1,1.3)$ & $0.4(0.1,1.2)$ & 0.09 \\
\hline \multicolumn{7}{|l|}{ Duration } \\
\hline$<7$ days & $0.2(0.1,0.4)$ & $0.2(0.1,0.4)$ & $<0.001$ & $0.5(0.2,1.0)$ & $0.5(0.2,1.0)$ & 0.06 \\
\hline 8 days -1 month & $1.8(0.5,6.1)$ & $2.1(0.6,7.8)$ & 0.27 & $3.3(1.1,90.0)$ & $4.3(1.1,16.4)$ & 0.04 \\
\hline $1-6$ months & $0.6(0.3,1.3)$ & $0.6(0.3,1.3)$ & 0.17 & $1.1(0.5,2.6)$ & $1.1(0.4,2.6)$ & 0.88 \\
\hline 6 months -1 year & $0.4(0.1,1.1)$ & $0.5(0.2,1.4)$ & 0.17 & $0.4(0.1,1.8)$ & $0.5(0.1,2.7)$ & 0.39 \\
\hline$>1$ year & 1.0 & 1.0 & - & 1.0 & 1.0 & - \\
\hline missing & $0.2(0.1,0.5)$ & $0.2(0.1,0.5)$ & $<0.001$ & $0.4(0.2,1.1)$ & $0.4(0.1,1.0)$ & 0.06 \\
\hline \multicolumn{7}{|l|}{ (b) St Mary's } \\
\hline Individuals & & & & & & \\
\hline Sex & & & & & & \\
\hline male & 1.0 & 1.0 & - & 1.0 & 1.0 & - \\
\hline female & $3.3(1.9,5.7)$ & $1.1(0.5,2.1)$ & 0.86 & $2.7(1.4,5.2)$ & $0.7(0.3,1.6)$ & 0.39 \\
\hline Orientation & & & & & & \\
\hline heterosexual & 1.0 & 1.0 & - & 1.0 & 1.0 & - \\
\hline homosexual & $0.3(0.2,0.5)$ & $0.5(0.2,1.0)$ & 0.06 & $0.2(0.08,0.4)$ & $0.2(0.08,0.6)$ & 0.003 \\
\hline Age group & & & & & & \\
\hline under 20 & 1.0 & 1.0 & - & 1.0 & 1.0 & - \\
\hline $20-25$ & $0.4(0.2,0.7)$ & $0.7(0.3,1.5)$ & 0.39 & $0.5(0.2,1.1)$ & $0.9(0.4,2.3)$ & 0.86 \\
\hline $25-30$ & $0.4(0.2,0.8)$ & $0.9(0.4,2.0)$ & 0.86 & $0.6(0.2,1.3)$ & $1.4(0.5,3.7)$ & 0.48 \\
\hline over 30 & $0.5(0.2,1.0)$ & $1.4(0.6,3.2)$ & 0.39 & $0.3(0.1,0.9)$ & $0.9(0.3,2.5)$ & 0.81 \\
\hline missing & $0.9(0.2,3.9)$ & $2.2(0.4,11.0)$ & 0.35 & $1.1(0.2,6.4)$ & $3.0(0.5,16.8)$ & 0.22 \\
\hline Ethnicity & & & & & & \\
\hline white & 1.0 & 1.0 & - & 1.0 & 1.0 & - \\
\hline black & $1.9(1.1,3.3)$ & $0.6(0.3,1.2)$ & 0.17 & $2.4(1.3,4.2)$ & $0.5(0.2,1.2)$ & 0.11 \\
\hline other & $0.5(0.1,2.1)$ & $0.4(0.1,1.4)$ & 0.16 & $0.4(0.04,3.0)$ & $0.2(0.03,1.2)$ & 0.14 \\
\hline missing & $0.6(0.2,1.9)$ & $0.5(0.2,1.5)$ & 0.23 & $0.2(0.03,1.9)$ & $0.2(0.02,1.1)$ & 0.07 \\
\hline No of partners & $0.9(0.8,1.0)$ & $1.0(0.9,1.0)$ & 0.24 & $0.9(0.8,1.0)$ & $1.0(0.9,1.1)$ & 0.51 \\
\hline Partnerships & & & & & & \\
\hline Type & & & & & & \\
\hline regular & 1.0 & 1.0 & - & 1.0 & 1.0 & - \\
\hline casual & $0.1(0.05,0.2)$ & $0.1(0.06,0.2)$ & $<0.001$ & $0.09(0.04,0.2)$ & $0.1(0.05,0.3)$ & $<0.001$ \\
\hline other & $0.2(0.06,0.6)$ & $0.2(0.05,0.6)$ & 0.01 & $0.2(0.04,0.8)$ & $0.2(0.03,1.1)$ & 0.07 \\
\hline missing & $0.04(0.01,0.2)$ & $0.1(0.02,0.6)$ & 0.01 & $0.05(0.02,0.4)$ & $0.3(0.04,1.6)$ & 0.15 \\
\hline Duration & & & & & & \\
\hline$<7$ days & $0.2(0.09,0.4)$ & $0.4(0.1,0.5)$ & $<0.001$ & $0.2(0.08,0.4)$ & $0.2(0.1,0.5)$ & $<0.001$ \\
\hline 8 days -1 month & $0.6(0.3,1.4)$ & $0.6(0.3,1.5)$ & 0.32 & $1.0(0.4,2.4)$ & $0.9(0.4,2.3)$ & 0.81 \\
\hline $1-6$ months & $0.7(0.3,1.3)$ & $0.7(0.4,1.4)$ & 0.36 & $0.3(0.1,0.9)$ & $0.3(0.1,0.9)$ & 0.02 \\
\hline 6 months -1 year & $1.3(0.7,2.6)$ & $1.4(0.7,2.9)$ & 0.40 & $1.1(0.5,2.6)$ & $1.0(0.4,2.3)$ & 0.95 \\
\hline$>1$ year & 1.0 & 1.0 & - & 1.0 & 1.0 & - \\
\hline missing & $0.2(0.07,0.3)$ & $0.3(0.1,0.7)$ & 0.005 & $0.2(0.06,0.4)$ & $0.3(0.1,0.9)$ & 0.03 \\
\hline
\end{tabular}

$\mathrm{OR}=$ odds ratio. Adjusted odds ratios significant at the $5 \%$ level in the multivariate analyses are shown in bold. Adjusted odds ratios for the demographics are shown from the model including partnership type; similar results were obtained in the model including partnership duration (results not shown). 
Table 2 Outcomes by partner notification agreement for outstanding contacts

\begin{tabular}{|c|c|c|c|c|c|c|}
\hline \multirow[b]{2}{*}{ Agreement reached } & \multicolumn{2}{|c|}{ First interview } & \multicolumn{2}{|c|}{ Final interview } & \multicolumn{2}{|c|}{ Contacts attending } \\
\hline & No & $\%$ & No & $\%$ & No & $\%$ \\
\hline \multicolumn{7}{|l|}{ Sheffield } \\
\hline Patient referral & $175 / 206$ & $85 \%$ & $95 / 169$ & $56 \%$ & $57 / 95$ & $60 \%$ \\
\hline Provider referral & $21 / 206$ & $15 \%$ & $74 / 169$ & $44 \%$ & $44 / 74$ & $59 \%$ \\
\hline All referrals & & & & & $101 / 169$ & $60 \%$ \\
\hline Contacts not sought, insufficient details & 453 & & 490 & & & \\
\hline \multicolumn{7}{|l|}{ St Mary's } \\
\hline Patient referral & $522 / 526$ & $99 \%$ & $446 / 450$ & $99 \%$ & $57 / 446$ & $13 \%$ \\
\hline Provider referral & $4 / 526$ & $1 \%$ & $4 / 450$ & $1 \%$ & $1 / 4$ & $25 \%$ \\
\hline All referrals & & & & & $58 / 450$ & $13 \%$ \\
\hline Contacts not sought, insufficient details & 1650 & & 1726 & & & \\
\hline
\end{tabular}

forms were fully or partially completed for $63 \%$ of contacts (486/768) in Sheffield and 52\% $(1182 / 2286)$ at St Mary's. The majority of contacts without data in Sheffield (260/282, 92\%) were the estimated number of clients of the 13 sex workers for whom data had not been sought. The majority of contacts without data at St Mary's (1021/1104, 92\%) were indicated by homosexual men reporting multiple partners who could not be discussed individually. In Sheffield there were 659 and at St Mary's 2176 outstanding contacts.

PARTNER NOTIFICATION OUTCOMES

The outcomes of partner notification for each clinic are shown in figure 1 . In both clinics only a minority of contacts were known to have been screened. The proportion of outstanding contacts screened was significantly greater in Sheffield $(19.6 \% v 4.5 \%, \mathrm{p}<0.001)$, although the figure for Sheffield is less reliable because the denominator includes an estimated number of sex clients. However, both the proportion of cases linked to another screened contact (74.9\% in Sheffield; $38.8 \%$ at St Mary's), and the proportion of cases linked to another case (58.7\% in Sheffield; $25.6 \%$ at St Mary's), were significantly greater in Sheffield $(\mathrm{p}<0.001$ in both cases).

PATIENT AND PARTNERSHIP CHARACTERISTICS Table 1 shows the association between the proportion of outstanding contacts screened and patient and partnership characteristics for Sheffield (a) and St Mary's (b). In univariate analyses, sex, sexual preference, age, ethnicity, partnership type, and partnership duration all influenced the partner notification outcome. However, in logistic regression analyses, many of the patient demographic characteristics were no longer significant, suggesting that these variables are associated with characteristics of the partnership.

In Sheffield, partners of female patients were less likely to attend as gonorrhoea positive contacts than those of male patients. At St Mary's, partners of homosexual or bisexual men were less likely to attend as gonorrhoea positive contacts.

Where relationship status was recorded, "casual" partners were significantly less likely to attend for screening, or attend as gonorrhoea positive contacts, than those classified as "regular". Contacts whose relationship status was missing were also significantly less likely to attend than regular partners in both settings.
Duration of partnership, which was highly correlated with relationship type, had a similar effect: outstanding contacts from partnerships which had lasted less than 7 days were less likely to attend for screening in either centre, or attend as gonorrhoea positive contacts at St Mary's. Casual outstanding contacts were significantly more likely to be screened in Sheffield than at St Mary's (odds ratio 10.6, CI 5.16-23.07).

REFERRAL METHOD

Table 2 shows that, by final interview, a referral method had been agreed for only a minority of outstanding contacts at both centres $(26 \%$ in Sheffield and $21 \%$ at St Mary's). Patient referral was the preferred method in both centres, although provider referral was selected for a much greater proportion of notifications in Sheffield than St Mary's (44\% v 1\%, p<0.001). More than half $(56 \%)$ of provider referrals in Sheffield were agreed during follow up interviews, which had been conducted with a larger proportion of cases in Sheffield (40\% $v 20 \%$, $\mathrm{p}<0.001)$. Overall, the proportion of referral agreements which resulted in verified contact attendance was greater in Sheffield (60\%) than at St Mary's $(13 \%)(p<0.001)$. The outcomes of patient and provider referral in Sheffield were comparable, at $60 \%$ and $59 \%$ attendance respectively $(p=0.90)$.

\section{Discussion}

Partner notification in both settings resulted in the recorded attendance of a minority of outstanding contacts. Previous studies reporting much higher proportions screened $(83 \%-92 \%)^{7-11}$ differed methodologically by excluding untraceable contacts and by including other infections. ${ }^{7810}$ Contacts who were unlikely to have been involved in transmission were excluded by other investigators, who used a shorter, ${ }^{10}$ more flexible, ${ }^{11}$ or unspecified contact period. ${ }^{7-9}$ The epidemiological significance of the high proportion of untraced outstanding contacts in this study is uncertain: many had a low risk of infection because exposure had been protected by condom use, or was likely to have occurred before the case patient became infected. However, the proportion of cases which were not linked to another diagnosed case $(41 \%$ in Sheffield; $74 \%$ at St Mary's) suggests that a substantial number of infected contacts were not traced in either setting. The limitation of partner notification as a control 
strategy rests upon the greater difficulty of tracing casual contacts, who may play an important role in continued transmission. ${ }^{4}$

Partner notification appears to have been more productive in the provincial setting of Sheffield. However, outcomes for St Mary's are likely to be underrepresented because local service organisation influenced the ease with which contact attendance could be verified. Contacts of cases in Sheffield were more likely to use the same clinic since there were no alternative services available in the city, and therefore attendance could usually be verified from in house records. By contrast, contacts of cases at St Mary had a choice of over 30 London services which did not centralise data, or consistently liaise with the referring clinic. ${ }^{1}$ This problem for St Mary's was compounded by fewer opportunities to reinterview patients to establish whether, and where, contacts had attended.

The more extensive use of provider referral in Sheffield made a significant additional contribution to outcomes by securing the attendance of contacts whom case patients had been unable or unwilling to notify. Even though a standardised policy made provider referral available in both settings, local conditions influenced the frequency with which such referrals could be agreed. The comparative stability of the population in Sheffield made it easier for health advisers to identify and locate contacts because casual liaisons were more frequently formed between local people who knew each other, and relatively reliable local records could be used to supplement partial information. Opportunities for health advisers to reinterview patients in Sheffield, which secured a majority of provider referral agreements, may have been facilitated by reattendance for deferred epidemiological treatment, a more efficient system of internal referral, and a higher ratio of staff and interview rooms. In Sheffield, the skills and confidence required to negotiate provider referral may have been enhanced by previous experience, and by an awareness of local effectiveness.

Partner notification outcomes, practice, and local conditions are clearly interrelated. Outcomes in any setting may be optimised by operational arrangements which facilitate adequate communication between patient and health adviser, and between clinics. Opportunities for further discussion with patients may be maximised by promoting reattendance and ensuring referral to an available health adviser, or by prearranged telephone follow up. A system is required to improve the accuracy of recorded outcomes for services in London: the viability of a centralised data exchange for metropolitan clinics requires further study.

In conclusion, the contribution made by partner notification to gonorrhoea control in both environments was limited by the high proportion of untraced contacts. Complementary screening and prevention initiatives are therefore required to target those at risk from casual relationships, including homosexual men and commercial sex workers. ${ }^{4}$ Despite these limitations, results from both settings demonstrate the case finding potential of partner notification, and results from Sheffield show the additional impact of provider referral.

An earlier analysis of these data was presented in October 1997 at the biannual international conference on STD and AIDS in Seville.

This work was supported by a grant from the NHS executive (North Thames)

S Day and A C Ghani were funded by The Wellcome Trust. We would like to thank the health advisers at Sheffield and St Mary's who recorded the data for this study.

Contributors: Gill Bell, main author, collection and analysis of data; Helen Ward, study design and management, preparation of manuscript; Sophie Day, study design and management, review of manuscript; Azra C Ghani, data management, statistical analysis, preparation of manuscript; Una Goan, collection and analysis of data, review of manuscript; Elizabeth Claydon, study design, supervision of data collection at St Mary's, review study design, supervision of data collection at St Mary's, review of manuscript; George Kinghorn, study design, supervision of
data collection in Sheffield, data analysis, preparation of manuscript.

1 Fitzgerald MR, Thirlby D, Bedford CA. The outcome of contact tracing for gonorrhoea in the UK. Int $\mathcal{A} S T D$ AIDS 1998; (in press).

2 Allen I, Hogg D. Work roles and responsibilities in genitourinary clinics. London: Institute for Policy Studies, 1993.

3 Cowan FM, French R, Johnson AM. The role and effectiveness of partner notification in STD control: a review. Genitourin Med 1996;72:247-52.

4 Day S, Ward H, Ghani AC, et al. Sexual networks and the control of gonorrhoea. Int $\mathcal{F}$ STD AIDS 1998; (in press).

5 Potterat JJ, Rothenburg RB, Woodhouse DE, et al. Gonorrhoea as a social disease. Sex Transm Dis 1985;12: 25-32.

6 Liang KL, Zeger SL. Longitudinal data analysis using generalized linear models. Biometrika 1986;73:13-22.

7 Burgess JA. A contact tracing procedure. $\mathrm{Br} \mathcal{F}$ Vener Dis 1963;39:113-17.

8 Wigfield AS. 27 years of uninterrupted contact tracing. The Tyneside scheme. Br f Vener Dis 1972;48:37-49.

9 Talbot MD; Kinghorn GR. Epidemiology and control of gonorrhoea in Sheffield. Genitourin Med 1985;61:230-3.

10 Katz B, Caine VA, Jones RB. Evaluation of field follow-up in sexually transmitted disease for patients at risk for infection with Neisseria gonorrhoeae and Chlamydia trachomatis. Sex Transm Dis 1992;19:99-104.

11 Ruden AK, Jonnson A, Lidbrink P, et al. Endemic versus non-endemic gonorrhoea in Stockholm: results of contacttracing. Int f STD AIDS 1993;4:284-22. 